\title{
Switching from contextual to tone fear conditioning and vice versa: The key role of the glutamatergic hippocampal-lateral septal neurotransmission
}

\author{
Ludovic Calandreau, ${ }^{1,2,3}$ Bertrand Desgranges, ${ }^{2}$ Robert Jaffard, ${ }^{1}$ and Aline Desmedt ${ }^{1}$ \\ ${ }^{1}$ Centre de Neurosciences Intégratives et Cognitives, CNRS UMR 5228, Université de Bordeaux, 33405 Talence, France; ${ }^{2}$ Physiologie de \\ la Reproduction et des Comportements, INRA UMR 85, CNRS UMR 6175, Université François Rabelais de Tours, Haras Nationaux, \\ 37380 Nouzilly, France
}

\begin{abstract}
The aim of the present experiment was to directly assess the role of the glutamatergic hippocampal-lateral septal (HPC-LS) neurotransmission in tone and contextual fear conditioning. We found that pretraining infusion of glutamatergic acid into the lateral septum promotes tone conditioning and concomitantly disrupts contextual conditioning. Infusion of glutamatergic antagonist, on the contrary, promotes contextual conditioning to the detriment of tone fear conditioning. These findings highlight the direct contribution of the glutamatergic HPC-LS neurotransmission to the adaptive selection among environmental stimuli of those that best predict the occurrence of the aversive event.
\end{abstract}

In Pavlovian fear conditioning, subjects adaptively select among environmental stimuli those that predict an aversive event (Fendt and Fanselow 1999; LeDoux 2000; Maren 2001; Paré et al. 2004; Yaniv et al. 2004). When a discrete conditioned stimulus (CS, a tone) is systematically paired with the unconditional stimulus (US, a footshock), subjects process this discrete CS as the main predictor and consign the less-predictive contextual cues to the background. In contrast, when the CS has no or low predictive value (e.g., CS-US unpairing), contextual cues are selected as valid predictors and processed in the foreground (Rescorla and Wagner 1972; Winocur et al. 1987; Desmedt et al. 1998, 1999; Calandreau et al. 2005, 2006, 2007). While neural bases of both tone and contextual fear conditioning have been extensively studied, surprisingly little is known regarding the mechanisms that specifically subserve the adaptive selection process of the simple tone CS or the context as the best predictive stimulus.

Using field potentials recordings, we previously showed that contextual fear conditioning was associated with a decrease in hippocampal-lateral septal (HPC-LS) synaptic transmission (Garcia and Jaffard 1996; Desmedt et al. 1998). In contrast, simple tone fear conditioning was associated with an increase in HPC-LS synaptic transmission, in particular when repeated CS-US pairings were used (Desmedt et al. 1998, 2003a). Moreover, vasopressin within the LS, which is thought to increase the glutamatergic HPC-LS transmission (Raggenbass et al. 1988; Van Den Hooff and Urban 1990), was required to promote the acquisition of tone fear conditioning, whereas vasopressin antagonist promoted contextual fear conditioning (Desmedt et al. 1999). Altogether, these data strongly suggest that changes in the glutamatergic HPC-LS neurotransmission might be critical for the selection of the most predictive CS for the occurrence of the aversive US. The aim of the present experiment was to directly assess the role of this neurotransmission in fear conditioning. Specifically, we tested the hypothesis that a pharmacologically induced increase in the glutamatergic transmission in the LS promotes elemental

\section{${ }^{3}$ Corresponding author.}

E-mail ludovic.calandreau@tours.inra.fr; fax 33247427743.

Article is online at http://www.learnmem.org/cgi/doi/10.1101//m.1859810. tone fear conditioning to the detriment of contextual conditioning, whereas blocking this transmission may lead to an inverse pattern of effects.

A total of 72 naïve adult (4- to 6-mo-old) male mice (C57Bl/6 JI Co, Charles River Laboratories, France) weighing 27-32 g were housed individually $7 \mathrm{~d}$ before all experiments and maintained in an animal room $\left(23^{\circ} \mathrm{C}\right)$ with a $12 \mathrm{~h}$ light/dark cycle $(07.00-$ $19.00 \mathrm{~h}$ lights on) with ad libitum access to food and water.

Mice were anesthesized with a solution of ketamine $(1 \mathrm{~mL}$; Merial) and xylazine $(0.1 \mathrm{~mL}$ in $8.9 \mathrm{~mL}$ water; Bayer $)(10 \mathrm{~mL}$ per kg body weight, injected intraperitoneally) and secured in a Kopf stereotaxic apparatus. Stainless-steel guide cannulae ( $0.45 \mathrm{~mm}$ in diameter, $8 \mathrm{~mm}$ length) aimed at the LS were implanted bilaterally $1 \mathrm{~mm}$ above the LS $(2.1 \mathrm{~mm}$ below the skull surface, $0.9 \mathrm{~mm}$ anterior to bregma, and $0.4 \mathrm{~mm}$ lateral from midline) (Franklin and Paxinos 1997), fixed in place with dental cement and two jewel screws attached to the skull. Animals were allowed to recover in their home cages in the animal room for at least $8 \mathrm{~d}$ before behavioral experiment.

The procedure and doses used for intraseptal infusion of glutamic acid and kynurenate were previously fully described and validated (Marighetto et al. 1994; Cazala et al. 1998; Desmedt et al. 1999; Calandreau et al. 2007). Briefly, immediately before the acquisition of fear conditioning, stainless-steel cannulae $(0.2 \mathrm{~mm}$ in diameter, $9 \mathrm{~mm}$ length) were inserted through the guides and each mouse was given bilateral infusion of saline $(0.9 \%)$, kynurenate $(\mathrm{KYN}$, a competitive antagonist at the glycine $_{\mathrm{B}}$ site of the NMDA receptor, $25 \mathrm{ng} / 1 \mu \mathrm{L}$, Sigma), or glutamic acid (GLU, $12.5 \mathrm{ng} / 1 \mu \mathrm{L}$, Sigma), into the LS over a 3-min period during which the animal was maintained in its home cage. In each hemisphere (each LS), a volume of $0.2 \mu \mathrm{L}$ of saline, kynurenate, or glutamic acid solution was infused.

Classical fear conditioning took place in a Plexiglas box $(30 \times$ $24 \times 22$-cm high) giving access to the visuo-spatial cues in the experimental room. The floor of this conditioning chamber consisted of stainless-steel rods (2-mm diameter), spaced 5-mm apart and connected to a shock generator (Imetronic, Talence, France). The four sides of the chamber and the rods of the floor were cleaned with $70 \%$ ethanol before each trial. For acquisition, half of the animals were submitted to two tone CS $(65 \mathrm{~dB}, 1 \mathrm{kHz}$, 
$15 \mathrm{sec})$-footshock US ( $0.7 \mathrm{~mA}, 50 \mathrm{~Hz}, 3 \mathrm{sec})$ pairings. In this training condition, the prevalent association is the elemental CS-US association, which consigns the conditioning context to the background. The other half was submitted to tone-shock unpairing: The only difference with the previous training condition was that the two shocks and the two tones were pseudo-randomly distributed. This explicit unpairing of CS and US is known to make the contextual cues the primary stimuli that enter into association with the US (i.e., context in the foreground) (Desmedt et al. 1999; Calandreau et al. 2005, 2006, 2007).

Twenty-four hours later, while mice were drug free, they were tested for freezing behavior (measured second-by-second), used as an index of conditioned fear and defined as a lack of all movement, except for respiratory-related movements (Fanselow 1980). First, all mice were submitted to the auditory cue test: They were maintained in their home cage and two successive recording sessions of behavioral responses were performed prior to (first $2 \mathrm{~min}$ ) and during (next $2 \mathrm{~min}$ ) tone presentation. Two hours later, mice were submitted to the context test: They were re-exposed to the conditioning chamber and two successive 2-min recordings of behavioral responses were performed. Animals were continuously videotaped for manual off-line scoring of freezing by an observer blind of experimental grouping. Freezing was calculated as the percentage $( \pm$ SEM) of the total time spent freezing during every 2 -min recording session.

After completion of the behavioral study, animals were given an overdose of sodium thiopental $(120 \mathrm{mg} / \mathrm{kg})$ and transcardially perfused with physiological saline, followed by $10 \%$ buffered formalin. Brains were post-fixed in formalin-saccharose 30\% solution for $1 \mathrm{wk}$, frozen, cut coronally on a sliding microtome into $50-\mu \mathrm{m}$ sections that were mounted on a gelatin-coated slide, and stained with thionine in order to evaluate the cannulae placements.

A representative guide cannulae placement and infusion site into the LS are shown in Figure 1. Among a total of 72 animals, nine mice were excluded from the analysis due to either dislocated guide cannulae placement relative to the targeted LS subregion or infusion sites that reached the lateral ventricle.

As illustrated in Figure 2, the training-dependent increase in freezing observed between the first and second block during the tone test in saline-infused mice trained with the pairing procedure, as compared with those trained with the unpairing procedure (block $\times$ conditioning interaction: $F_{(1,18)}=29.63, P<$ 0.0001) was totally abolished in GLU- and KYN-infused animals (in GLU and KYN: block $\times$ conditioning, Fs $<1$ ). The pharmacological-induced disruption of the training-dependent increase of freezing to the tone was confirmed by a significant
A

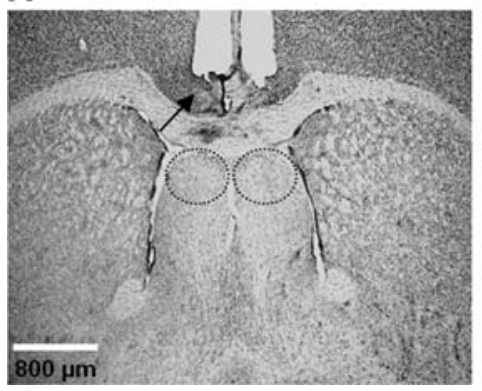

B

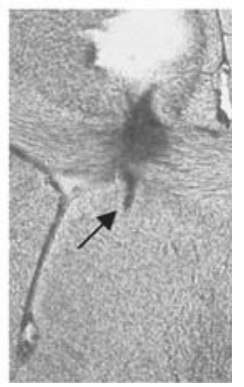

Figure 1. (A) Representative guide cannulae placement $1 \mathrm{~mm}$ above the dorsal lateral septum (arrow) and infusion sites (circles) into the lateral septum. (B) Representative photomicrograph of the exact location of the delivery site of drugs (arrow) within the dorsal the lateral septum.

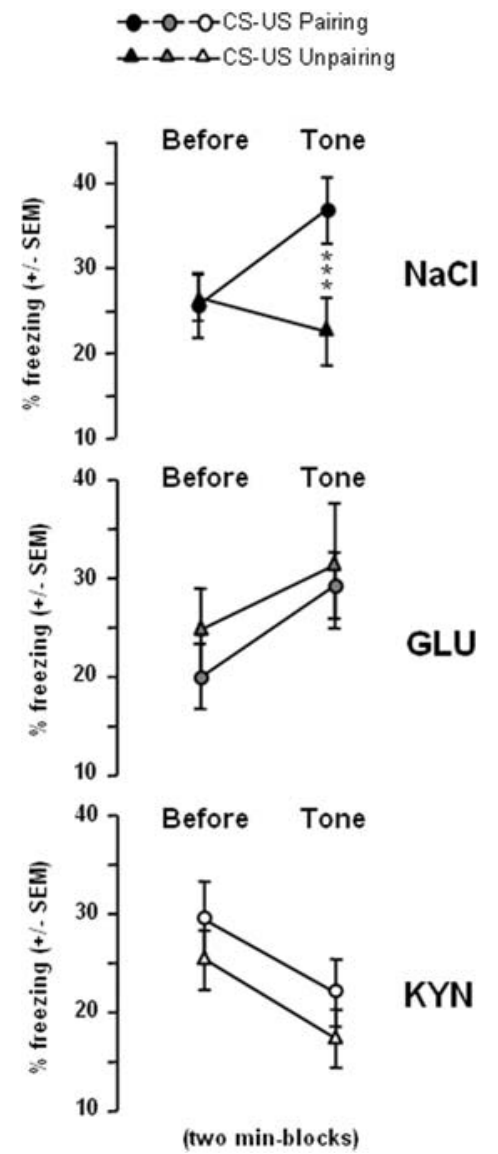

Figure 2. Auditory cue test. Mean percentage freezing ( \pm SEM) on successive 2-min blocks, before (first block) and during (second block) tone presentation, in animals that received $\mathrm{NaCl}(n=20)$, glutamic acid (GLU, $n=21$ ), or kynurenate (KYN, $n=22$ ) into the dorsal lateral septum before acquisition with either a CS-US pairing or unpairing conditioning procedure. $\left({ }^{* *}\right)$ Statistically significant block $\times$ conditioning procedure interaction: $P<0.0001$.

block $\times$ conditioning $\times$ treatment interaction (GLU vs. $\mathrm{NaCl}$ : $F_{(1,37)}=4.40, P=0.042 ; \mathrm{KYN}$ vs. NaCl: $\left.F_{(1,38)}=6.08, P=0.018\right)$. Specifically, intra-LS infusions of glutamic acid and kynurenate had opposite effects on conditioned freezing to the discrete tone. Under both conditioning procedures, GLU-infused animals displayed a significant increase of conditioned freezing during tone presentation as compared with before (block effect: $\left.F_{(1,19)}=8.70, P=0.008\right)$. Conversely, KYN-infused animals displayed significantly less-conditioned freezing during tone presentation than before $\left(F_{(1,20)}=9.65, P=0.005\right)$, whatever the conditioning procedure used. These training- and treatmentdependent differences in conditioned freezing to the tone cannot be explained by general effects of the training or treatment on the freezing expression per se. Indeed, a restricted analysis performed on the first 2-min block preceding the occurrence of the tone revealed that animals displayed similar levels of freezing with no significant effect of the training procedure $\left(F_{(1,57)}=0.026\right.$; ns), nor of the treatment $\left(F_{(2,57)}=0.91 ; n s\right)$.

Figure 3 shows that, during the context test, saline-infused mice trained under the unpairing procedure (context in the foreground) displayed significantly more conditioned freezing to the context than saline-infused mice trained with the pairing procedure (context in the background). This training-dependent effect 


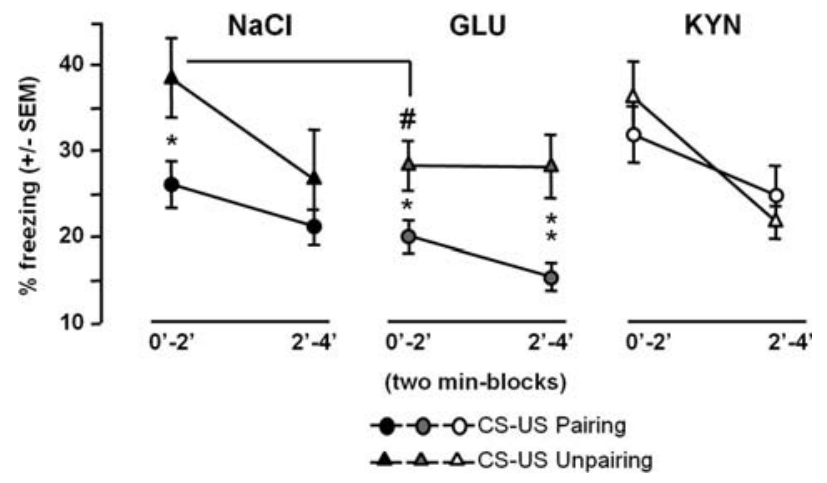

Figure 3. Context test. Mean percentage freezing ( \pm SEM) on two successive 2-min blocks during re-exposure to the conditioning context in animals that received $\mathrm{NaCl}$, glutamic acid (GLU) or kynurenate (KYN) infusions into the dorsal lateral septum before acquisition with either a CS-US pairing or unpairing procedure. (*) Statistically significant conditioning effect (CS-US pairing vs. unpairing): $P<0.05$; (\#) treatment effect (GLU vs. $\mathrm{NaCl}$ ): $P<0.05$.

on contextual conditioning was especially observed during the first 2-min block $\left(F_{(1,18)}=5.35, P=0.032\right)$. GLU-infused mice also displayed this training-dependent effect as they displayed more conditioned freezing to the context in the unpairing than in the pairing procedure $\left(F_{(1,19)}=5.82, P=0.026\right)$ even during the second 2-min block $\left(F_{(1,19)}=10.42, P=0.004\right)$. However, they overall displayed significantly lower levels of conditioned freezing to the context than saline-infused mice (effect of treatment, GLU vs. NaCl: $\left.F_{(1,37)}=6.75, P=0.013\right)$, irrespective of the conditioning procedure used (treatment $\times$ conditioning: $\left.F_{(1,37)}=0.44 ; \mathrm{ns}\right)$. In contrast, the training-dependent effect on contextual freezing was completely abolished in KYN-infused mice $\left(F_{(1,20)}=0.73 ; \mathrm{ns}\right)$, which, nevertheless, displayed substantial levels of freezing that did not significantly differ from that of saline-infused mice (effect of treatment $\mathrm{KYN}$ vs. $\mathrm{NaCl}$ : $F_{(1,38)}=0.21$; ns) whatever the conditioning procedure used (treatment $\times$ conditioning: $F_{(1,38)}=1.11$; ns).

The differential effect of intra-LS infusions of the glutamatergic agonist and antagonist on tone and contextual fear conditioning are highly congruent with previous electrophysiological, lesioning, and pharmacological studies. Using field potentials recordings, we previously showed that a decrease in the HPC-LS glutamatergic neurotransmission was associated with preferential conditioning to contextual cues (CS-US unpairing procedure). Moreover, induction of long-term potentiation (LTP) in the LS by fimbrial high-frequency stimulation was shown to produce an impairment of conditioned freezing to contextual cues, whereas lesions of LS neurons underlying this LTP strongly reduced this impairing effect (Vouimba et al. 1998). In contrast, an increase in this HPC-LS neurotransmission was associated with tone fear conditioning (CS-US pairing), in particular when extensive tone CS-US pairings were used (Garcia and Jaffard 1996; Desmedt et al. 1998, 2003a). In addition, we recently showed that pretraining inactivation of the LS disrupted tone fear conditioning, while sparing fear conditioning to the foreground context (Calandreau et al. 2007). Altogether, these results provided evidence that increasing levels of the HPC-LS neurotransmission promotes conditioning to an elemental (tone) cue, whereas decreasing levels of this neurotransmission promotes conditioning to contextual cues.

In line with this, a pharmacological study has previously shown that pretraining intra-LS infusions of vasopressin, which is known both to increase HPC-LS glutamatergic neurotransmission and to facilitate the maintenance of LTP in the LS (Raggenbass et al. 1988; Van Den Hooff et al. 1989; Van Den Hooff and Urban 1990), not only spared fear conditioning to a tone CS that was fully predictive for the occurrence of the US (CS-US pairing), but promoted such elemental conditioning when the tone CS was not predictive for the occurrence of the US (CS-US unpairing). Concomitantly, infusion of vasopressin into the LS was associated with an impairment of contextual fear conditioning. An opposite pattern of effects was observed using vasopressinergic antagonists (Desmedt et al. 1999). In agreement with these previous data, our present findings actually provide direct evidence that increasing and blocking the glutamatergic HPC-LS neurotransmission (1) promotes and blocks the acquisition of elemental tone fear conditioning, respectively, and (2) concomitantly produces an opposite pattern of effects on contextual fear conditioning. Altogether, these findings indicate that the glutamatergic HPC-LS neurotransmission regulates both adaptive tone and contextual fear conditioning.

It is unlikely that drug injections have unspecific effects on locomotor activity and indirectly modulate freezing behavior. Indeed, injections were assessed immediately before training. Consequently, when the auditory cue test and the context test were performed $24 \mathrm{~h}$ later, animals were drug free. Moreover, an unspecific effect of the drugs would have resulted in general increased or decreased freezing levels. In the present study, each treatment was associated with opposite effects on simple tone CS and contextual freezing behaviors. Finally, saline and drug-injected mice did not differ in baseline freezing behavior, confirming the idea of an absence of an unspecific direct or indirect effect of the drug on freezing behavior.

Rather, the reported bidirectional changes in HPC-LS synaptic excitability as a function of the training conditioning procedure (CS-US pairing vs. unpairing) (Garcia and Jaffard 1996; Desmedt et al. 1998, 2003a) provides evidence that levels of the HPC-LS glutamatergic transmission causally contribute to the selection of the tone CS as either predictive or nonpredictive of the aversive US. Thus, blocking this neurotransmission totally abolished the acquisition of the tone CS-US association (CS-US pairing). Conversely, increasing this neurotransmission was sufficient to produce conditioned fear to the nonpredictive (CS-US unpairing) discrete tone CS. It must be noted that the tone, although not predictive of the occurrence of the US in this training condition, remains the only phasic simple stimulus and, accordingly, would constitute a more salient stimulus than the entire set of static stimuli that make up the context. Thus, changes in the HPC-LS glutamatergic neurotransmission may primarily act on the selection of the CS as a function of its saliency rather than on its predictive value for the occurrence of the US (see Desmedt et al. 1999).

While sparing (CS-US pairing) or promoting (CS-US unpairing) tone fear conditioning, intra-LS infusions of glutamatergic agonist impaired levels of contextual freezing in both training conditions. Conversely, intra-LS infusions of glutamatergic antagonist spared substantial levels of contextual freezing in both training conditions. Since contextual fear conditioning has been shown to require the functional integrity of the hippocampus (Selden et al. 1991; Desmedt et al. 2003b; Paré et al. 2004; Calandreau et al. 2006), the present finding suggests that manipulating the HPC-LS glutamatergic neurotransmission interferes with the hippocampal-dependent processing of contextual cues. It is assumed that glutamatergic receptors localized in the LS exert, via an increase in GABAergic cells excitability, an inhibitory effect on cholinergic cells in the medial septum/ diagonal band of Broca (Giovannini et al. 1994; Marighetto et al. 1994). Consequently, increasing the HPC-LS glutamatergic 
neurotransmission would impair the hippocampal (cholinergic signal)-dependent processing of contextual cues (see Desmedt et al. 1999), and thus, contextual conditioning. Conversely, decreasing the HPC-LS glutamatergic neurotransmission would enhance the processing of contextual cues by the hippocampus, thereby resulting in substantial levels of contextual conditioned freezing. In accordance with this assumption, we recently showed that pretraining inactivation of the medial septum (MS), which constitutes the predominant source of hippocampal cholinergic input, selectively interferes with the establishment of the appropriate level of contextual conditioned freezing as a function of the training procedure (Calandreau et al. 2007). Moreover, using microdialysis, we showed that the level of contextual fear conditioning was directly related to the level of the cholinergic release measured in the hippocampus. In particular, foreground contextual fear conditioning (CS-US unpairing) was shown to be associated with a higher hippocampal cholinergic release than background contextual fear conditioning (CS-US pairing). Finally, we recently demonstrated that increasing or decreasing hippocampal cholinergic neurotransmission with intrahippocampal infusions of physostigmine or scopolamine mimicked foreground and background contextual fear conditioning, respectively (Calandreau et al. 2006).

In conclusion, the present study highlights the direct contribution of the glutamatergic HPC-LS neurotransmission to the acquisition of adaptive conditioned fear responses as a function of the learning situation. Our suggestion is that LS glutamate levels play a critical role in the selection of relevant stimuli, thereby contributing to the adaptive value of emotional memory rather than to the absolute level of conditioned fear expressed per se. Since this ability to appropriately select or process relevant emotional information is altered in several trauma-related pathologies, such findings might highlight neural dysfunctions involved in pathological emotional memories.

\section{Acknowledgments}

We thank A. Marighetto and P. Trifilieff for helpful comments on the manuscript. This study was supported by the Centre National de la Recherche Scientifique and by the Fondation pour la Recherche sur le Cerveau (FRC).

\section{References}

Calandreau L, Desmedt A, Decortes L, Jaffard R. 2005. A different recruitment of the lateral and basolateral amygdala promotes contextual or elemental conditioned association in Pavlovian fear conditioning. Learn Mem 12: 383-388.

Calandreau L, Trifilieff P, Mons N, Costes L, Marien M, Marighetto A, Micheau J, Jaffard R, Desmedt A. 2006. Extracellular hippocampal acetylcholine level controls amygdala function and promotes adaptive conditioned emotional response. J Neurosci 26: 13556-13566.

Calandreau L, Jaffard R, Desmedt A. 2007. Dissociated roles for the lateral and medial septum in elemental and contextual fear conditioning. Learn Mem 14: 422-429.

Cazala P, Norena A, Le Merrer J, Galey D. 1998. Differential involvement of the lateral and medial divisions of the septal area on spatial learning processes as revealed by intracranial self-administration of morphine in mice. Behav Brain Res 97: 179-188.

Desmedt A, Garcia R, Jaffard R. 1998. Differential modulation of changes in hippocampal-septal synaptic excitability by the amygdala as a function of either elemental or contextual fear conditioning in mice. J Neurosci 18: $480-487$.

Desmedt A, Garcia R, Jaffard R. 1999. Vasopressin in the septum promotes elemental conditioning to the detriment of contextual fear conditioning in mice. Eur J Neurosci 11: 3913-3921.

Desmedt A, Garcia R, Jaffard R. 2003a. An 8-day extensive elemental, but not contextual, fear conditioning potentiates hippocampallateral septal synaptic efficacy in mice. Synapse 49: 270-278.

Desmedt A, Marighetto A, Garcia R, Jaffard R. 2003b. The effects of ibotenic hippocampal lesions on discriminative fear conditioning in mice: Impairment or facilitation depending of the associate value of a phasic explicit cue. Eur J Neurosci 17: 1953-1963.

Fanselow MS. 1980. Conditional and unconditional components of post-shock freezing. Pavlov J Biol Sci 15: 177-182.

Fendt M, Fanselow MS. 1999. The neuroanatomical and neurochemical basis of conditioned fear. Neurosci Biobehav Rev 23: $743-760$

Franklin KBJ, Paxinos G. 1997. The mouse brain in stereotaxic coordinates. Academic Press, New York.

Garcia R, Jaffard R. 1996. Changes in synaptic excitability in the lateral septum associated with contextual and auditory fear conditioning in mice. Eur J Neurosci 8: 809-815.

Giovannini MG, Mutolo D, Bianchi L, Michelassi A, Pepeu G. 1994. NMDA receptor antagonists decrease GABA outflow from the septum and increase acetylcholine outflow from the hippocampus: A microdialysis study. J Neurosci 14: 1358-1365.

LeDoux JE. 2000. Emotion circuits in the brain. Annu Rev Neurosci 23: $155-184$.

Maren S. 2001. Neurobiology of Pavlovian fear conditioning. Annu Rev Neurosci 24: 897-931.

Marighetto A, Micheau J, Jaffard R. 1994. Effects of intraseptally injected glutamatergic drugs on hippocampal sodium-dependant high-affinity choline uptake in "naïve" and "trained" mice. Pharmacol Biochem Behav 49: 689-699.

Paré D, Quirk GJ, Ledoux JE. 2004. New vistas on amygdala networks in conditioned fear. J Neurophysiol 92: 1-9.

Raggenbass M, Dubois-Dauphin M, Tribollet E, Dreifuss JJ. 1988. Direct excitatory action of vasopressin in the lateral septum of the rat brain. Brain Res 459: 60-69.

Rescorla RA, Wagner AR. 1972. A theory of Pavlovian conditioning: Variations in the effectiveness of reinforcement and non reinforcement. In Classical conditioning II (ed. AH Black, WF Prokasy), pp. 64-99. Appleton-Century-Croft, New York.

Selden NR, Everitt BJ, Jarrard LE, Robbins TW. 1991. Complementary roles for the amygdala and hippocampus in aversive conditioning to explicit and contextual cues. Neuroscience 42: 335-350.

Van den Hooff P, Urban IJ. 1990. Vasopressin facilitates excitatory transmission in slices of the rat dorso-lateral septum. Synapse 5: 201-206.

Van den Hooff P, Urban IJ, de Wied D. 1989. Vasopressin maintains long-term potentiation in rat lateral septum slices. Brain Res 505: $181-186$.

Vouimba RM, Garcia R, Jaffard R. 1998. Opposite effects of lateral septal LTP and lateral septal lesions on contextual fear conditioning in mice. Behav Neurosci 112: 875-884.

Winocur G, Rawlins JN, Gray JA. 1987. The hippocampus and conditioning to contextual cues. Behav Neurosci 101: 617-625.

Yaniv D, Desmedt A, Jaffard R, Richter-Levin G. 2004. The amygdala and appraisal processes: Stimulus and response complexity as an organizing factor. Brain Research Rev 44: 179-186

Received May 5, 2010; accepted in revised form July 21, 2010. 


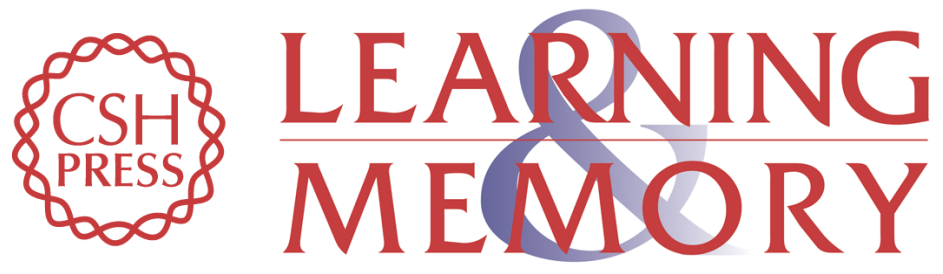

\section{Switching from contextual to tone fear conditioning and vice versa: The key role of the glutamatergic hippocampal-lateral septal neurotransmission}

Ludovic Calandreau, Bertrand Desgranges, Robert Jaffard, et al.

Learn. Mem. 2010, 17:

Access the most recent version at doi:10.1101//m.1859810

References This article cites 23 articles, 5 of which can be accessed free at: http://learnmem.cshlp.org/content/17/9/440.full.html\#ref-list-1

License

Email Alerting

Service

Receive free email alerts when new articles cite this article - sign up in the box at the top right corner of the article or click here. 